PROCEEDINGS OF THE

AMERICAN MATHEMATICAL SOCIETY

Volume 127, Number 2, February 1999, Pages 397-399

S 0002-9939(99)04929-1

\title{
A COMPLEX MAP WITH COMPLEX TOPOLOGY
}

\author{
MICHAŁ KWIECIŃSKI
}

(Communicated by Leslie D. Saper)

\begin{abstract}
We construct a simple example of a complex algebraic map, which (even locally) has an uncountable number of local topological types at points of the source space.
\end{abstract}

Let $f: X \rightarrow Y$ be a morphism of complex algebraic varieties. We say that $f$ has the same local topological type at two points $a$ and $b$ of $X$, if there exist homeomorphisms of germs (in the strong topology) $\phi: X_{a} \rightarrow X_{b}$ and $\psi: Y_{f(a)} \rightarrow$ $Y_{f(b)}$, such that $\psi \circ f_{a}=f_{b} \circ \phi$.

The aim of this note is to provide a simple example of a complex algebraic map $f: X \rightarrow Y$ which (even locally) has an infinite number of different local topological types at points of $X$. This contrasts with the topology of complex spaces, where by the theory of Whitney stratifications one has a locally finite number of local topological types. Also, if $Y$ is a smooth curve, then $f$ can be stratified to verify Thom's $a_{f}$ condition [2] which implies local finiteness.

There are related examples of Thom [6] and Nakai [4]. The difference with ours is that they treat the varying of topological type in parametrized families of maps. Nevertheless, our example is inspired by Thom's (which is real and global).

First, we introduce a tool, which is a concrete realization of an idea of Thom. ${ }^{1}$ On each fibre $f^{-1}(y)$, we define an invariant relation $\mathcal{R}(y)$ (a subset of $f^{-1}(y) \times$ $\left.f^{-1}(y)\right)$. Let $U=\left\{x \in X: \operatorname{dim}_{x} f^{-1}(f(x))=\operatorname{dim}_{x} X-\operatorname{dim}_{f(x)} Y\right\}$.

Definition 1. For $y \in Y$, let $\mathcal{R}(y)=f^{-1}(y) \times f^{-1}(y) \cap$ closure of $(U \times \underset{Y}{\times} U)$, where the closure is taken in the fibred product $X \underset{Y}{\times} X$ induced by the map $f$.

Definition 2. Let $A$ and $B$ be topological spaces, $a \in A, b \in B$. Let $\rho$ and $\delta$ be relations on $A$ and $B$, respectively. We say that $(\rho, a)$ and $(\delta, b)$ are topologically equivalent if there exists a homeomorphism of germs $g: A_{a} \rightarrow B_{b}$, such that in the induced product homeomorphism $g \times g:(A \times A)_{(a, a)} \rightarrow(B \times B)_{(b, b)}$ we have $(g \times g)^{-1}\left(\delta_{(b, b)}\right)=\rho_{(a, a)}$.

Proposition 1. If $f$ has the same local topological type at two points a and $b$ of $X$, then $(\mathcal{R}(f(a)), a)$ and $(\mathcal{R}(f(b)), b)$ are topologically equivalent. (Here the relations are considered on the fibres $A=f^{-1}(f(a))$ and $\left.B=f^{-1}(f(b))\right)$.

Received by the editors June 3, 1997

1991 Mathematics Subject Classification. Primary 14E40; Secondary 32S15.

The author's research was supported by a postdoctoral fellowship of the Japan Society for the Promotion of Science and Monbusho grant (number P96204).

${ }^{1}$ In [6] Thom writes “...dans un voisinage d'une strate éclatée, l'application opère des identifications qui se traduisent par une correspondance dans la strate (S) elle-même;...".

(C)1999 American Mathematical Society 
Since Definition 1 involves only local topological objects, Proposition 1 is obvious.

Example. Let $X$ be the hypersurface $x_{1} x_{2}=0$ in $\mathbb{C}^{4}$ with variables $\left(x_{1}, x_{2}, z, t\right)$. Let $Y=\mathbb{C}^{3}$, and define the map $f: X \rightarrow Y$ by

$$
f\left(x_{1}, x_{2}, z, t\right)=\left(x_{1}+x_{2},\left(x_{1}+t x_{2}\right) z, t\right) .
$$

Claim. In any neighbourhood of any point $(0,0,0, t) \in X$, with $|t|=1$, the map $f$ has infinitely many different local topological types. More precisely, suppose that $\alpha_{1}, \alpha_{2} \in[0,1), \alpha_{2}$ is irrational, $\alpha_{1} \neq \alpha_{2}$ and $\alpha_{1} \neq 1-\alpha_{2}$. Let $t_{1}=e^{2 \pi \alpha_{1} i}$ and $t_{2}=e^{2 \pi \alpha_{2} i}$. Then $f$ has different local topological types at $\left(0,0,0, t_{1}\right)$ and at $\left(0,0,0, t_{2}\right)$.

Proof of the claim. For $t \in \mathbb{C}$, write $a_{t}=(0,0,0, t)$. We first determine the invariant relation $\mathcal{R}\left(f\left(a_{t}\right)\right)$. Notice that $f^{-1}\left(f\left(a_{t}\right)\right) \cong \mathbb{C}$, with coordinate $z$. Let $\left(z, z^{\prime}\right)$ be corresponding coordinates in $f^{-1}\left(f\left(a_{t}\right)\right) \times f^{-1}\left(f\left(a_{t}\right)\right) \cong \mathbb{C}^{2}$. Then, the relation $\mathcal{R}\left(f\left(a_{t}\right)\right)$ is the hypersurface $H_{t}\left(z, z^{\prime}\right)=0$, with

$$
H_{t}\left(z, z^{\prime}\right)=\left(z-z^{\prime}\right)\left(z-t z^{\prime}\right)\left(t z-z^{\prime}\right) .
$$

To establish this, remark that the set $U$ from Definition 1 is in this case the complement of $\left\{x_{1}=x_{2}=0\right\} \cup\left\{t=x_{1}=0\right\}$. Then, one can use computer algebra to calculate the appropriate closure or do it directly (look at limits of pairs of points of $U$ with the same values of $f$ ). We leave these simple details to the reader.

By Proposition 1 it is enough to show that $\left(\mathcal{R}\left(f\left(a_{t_{1}}\right)\right), 0\right)$ and $\left(\mathcal{R}\left(f\left(a_{t_{2}}\right)\right), 0\right)$ are not topologically equivalent. Assume the contrary. This means that there exists a homeomorphism of germs $g: \mathbb{C}_{0} \rightarrow \mathbb{C}_{0}$, such that the product homeomorphism $g \times g: \mathbb{C}_{0}^{2} \rightarrow \mathbb{C}_{0}^{2}$ maps the germ of the hypersurface $H_{t_{1}}\left(z, z^{\prime}\right)=0$ to the germ of $H_{t_{2}}\left(z, z^{\prime}\right)=0$. Of course, the diagonal is mapped to the diagonal and every other irreducible component into an irreducible component (since after removing the origin they become connected components). Suppose that the component $z^{\prime}=t_{1} z$ is mapped to $z^{\prime}=t_{2} z$ (the other case being similar). This means that $g$ satisfies the following identity on $\mathbb{C}_{0}$ :

$$
e^{2 \pi \alpha_{1} i} z=g^{-1}\left(e^{2 \pi \alpha_{2} i} g(z)\right) .
$$

Replace $g$ by a representative and let $S$ be a small circle centered at 0 . Now $S$ is closed under multiplication by $t_{1}$ and, therefore, $g(S)$ is closed under multiplication by $t_{2}$. Since $\alpha_{2}$ is irrational, $g(S)$ is also a circle. Thus, the above identity holds after restricting $g$ and $g^{-1}$ to circles. This implies that rotations of the circle by $2 \pi \alpha_{1}$ and by $2 \pi \alpha_{2}$ have the same rotation number (see e.g. [1],[3],[5]) and contradicts the assumption that $\alpha_{1} \neq \alpha_{2}$.

\section{REFERENCES}

[1] V.I. Arnold, Geometrical methods in the theory of ordinary differential equations, Springer Grundlehren der mathematischen Wissenschaften 250, 1988. MR 89h:58049

[2] H. Hironaka, Stratification and Flatness, in Real and Complex Singularities, Proc. Oslo 1976, ed. Per Holm, Stijthof and Noordhof 1997, 199-265. MR 58:17187

[3] E.R. van Kampen, The topological transformations of a simple closed curve into itself, American Journal of Mathematics 57 (1935), 142-152.

[4] I. Nakai, On topological types of polynomial mappings, Topology 23 (1984), 45-66. MR 85g:58076 
[5] J. Palis, W. de Melo, Geometric theory of dynamical systems, Springer, New York 1982. MR 84a:58004

[6] R. Thom, La stabilité topologique des applications polynomiales, Enseign. Math. 8 (1962), 24-33. MR 26:5588

Department of Mathematics, Tokyo Metropolitan University, Minami - Ohsawa 1-1, HACHIOJI-SHI, TOKYO 192-03, JAPAN

On leave from: Uniwersytet Jagielloński, Instytut Matematyki, ul. Reymonta 4, 30059 Kraków, Poland

E-mail address: kwiecins@im.uj.edu.pl

URL: http://www.im.uj.edu.pl/ kwiecins 